\title{
Microtubule Stability Decreases Axon Elongation but Not Axoplasm Production
}

\author{
M. William Rochlin, ${ }^{1}$ Karen M. Wickline, ${ }^{2}$ and Paul C. Bridgman ${ }^{1}$ \\ 1 Department of Anatomy and Neurobiology, Washington University School of Medicine, and 2Department of Pediatrics, \\ St. Louis Children's Hospital, St. Louis, Missouri 63110
}

\begin{abstract}
Microtubules are a primary cytoskeletal constituent of axons and growth cones. In addition to serving as a scaffolding for axon assembly, they also provide a means of transport of organelles that are essential for outgrowth and maintenance of synaptic function. Pharmacological manipulations that disrupt net assembly of microtubules also interfere with growth cone advance and axon extension. Less is known about the effects of disrupting microtubule dynamics without affecting net assembly. To investigate this, we studied the effects of low doses of nocodazole on axon extension and microtubule organization in rat superior cervical ganglion neurons. We report that $165-$ $330 \mathrm{~nm}$ nocodazole significantly reduces axon extension rate and increases axon diameter without affecting the rate of production of axoplasm or microtubule polymer, and without decreasing the average length or number of microtubules. Two
\end{abstract}

Microtubules are one of the main cytoskeletal components of axons and are necessary for axonal outgrowth under normal physiological conditions. There has been considerable debate over possible mechanisms underlying the formation of the axonal microtubule network. Two main models exist for its construction. In one model, all microtubules are nucleated by the microtubule organizing center (MTOC) in the cell body, and after a short period of growth are released and transported into the axon with their plus ends toward the growth cone (Baas and Ahmad, 1993; Joshi and Baas, 1993; Ahmad and Baas, 1995). In an alternative model, microtubules are essentially stationary in the axon, and new microtubules form near the growth cone (Lim et al., 1990; Hirokawa, 1993). Both models emphasize the importance of microtubule assembly (at different sites) and the requirement for continued growth of axonal microtubules. Neither model addresses the potential importance of the dynamic instability of microtubules to assembly of the microtubule network and, more generally, to assembly of the axon.

Dynamic instability refers to the stochastic process of assembly and disassembly of microtubule plus ends (Mitchison and Kirshner, 1984). At steady state, the plus ends of microtubules undergo variable periods of assembly, disassembly, or pauses in assembly.

\footnotetext{
Reccived Nov. 1, 1995; revised Fcb. 21, 1996; accepted Feb. 26, 1996.

This work was supported by grants from National Institutes of Health to P.B. We thank Grady Phillips for expert help with the electron microscopy. We are grateful to Lisa Evans, and Drs. Bill Zaks and Soo-Siang Lim for comments on this manuscript.

Correspondence should be addressed to Dr. Paul C. Bridgman, Department of Anatomy and Neurobiology, Washington Universily School of Medicine, 660 South Euclid Avenue, St. Louis, MO 63110.

M.W.R. and K.M.W. contributed equally to this study.

Copyright $C 1996$ Society for Neuroscience $0270-6474 / 96 / 163236-11 \$ 05.00 / 0$
}

observations suggested that microtubule dynamics were depressed by this dose of nocodazole. First, this treatment eliminated the highly divergent lengths and positions of microtubules characteristic of normal growth cones, inducing an array in which each microtubule terminated at roughly the same position in the proximal regions of the growth cone. Second, there was a decrease in the proportion of microtubule length containing mostly tyrosinated (newly assembled) $\alpha$-tubulin and an increase in the proportion of microtubule length containing mostly acetylated (older, more stable) $\alpha$-tubulin. Together, these data suggest that a decrease in dynamic instability of microtubules is sufficient to disrupt axon extension but does not interfere with axoplasm production.

Key words: microtubule; axon; polymerization; nocodazole; dynamic instability; growth cone
The minus ends of microtubules appear to be relatively stable, particularly in axons (Baas and Black, 1990). The difference in stability between the two ends results in part from the intrinsic properties of polymerized tubulin. In addition, the kinetic properties of microtubules are regulated by microtubule associated proteins and association with the axonal cytoskeleton (Hirokawa, 1994). Tubulin in axonal microtubules can be post-translationally modified (detyrosinated or acetylated) (Brown et al., 1990). In vivo, these modifications are correlated with, but do not appear to cause, an increase in the stability of the microtubules to destabilizing treatments (Gelfand and Bershadsky, 1991; Li and Black, 1996). The degree of regulation of microtubule assembly kinetics in axons suggests that this highly variable property, rather than simply net polymerization, is an important parameter in how microtubules participate in the cytoskeletal remodeling that underlies axon assembly.

We investigated the consequences of decreasing the variability of microtubule plus end assembly kinetics by using low doses of nocodazole (Jordan and Wilson, 1990; Jordan et al., 1992). Disruption of microtubule dynamics with low doses of vinblastine perturbs normal forward translocation of the growth cone and the formation of new axon (Tanaka et al., 1995), and low doses of nocodazole disrupt fibroblast locomotion (Liao et al., 1995). However, low doses of vinblastine, in addition to decreasing the dynamics of plus end assembly, decrease net microtubule production (Baas and Ahmad, 1993). Nocodazole is less toxic than vinblastine (De Brabander et al., 1976) and does not inhibit net microtubule assembly rates. We report that low doses of nocodazole also decreased growth cone advance rates. Because our results indicate that nocodazole does not have deleterious effects on neuronal health or the production of axoplasm or microtu- 
Table 1. Axon volume and microtubule mass

\begin{tabular}{|c|c|c|c|c|c|c|c|}
\hline Treatment & $\begin{array}{l}\text { Axon diameter } \\
(\mu \mathrm{m})( \pm \mathrm{SEM})\end{array}$ & $\begin{array}{l}\text { Axon length }(\mu \mathrm{m}) \\
( \pm \text { SEM }), \# \text { cells }\end{array}$ & $\begin{array}{l}\text { Axon } \\
\text { volume } \\
\left(\mu \mathrm{m}^{3}\right)\end{array}$ & $\begin{array}{l}\text { Corrected axon } \\
\text { volume } \mathrm{c}^{b}\left(\mu \mathrm{m}^{3}\right)\end{array}$ & $\begin{array}{l}\text { Microtubules per } \\
\text { cross-section } \\
( \pm \text { SEM }), \# \\
\text { cross-section profiles }\end{array}$ & $\begin{array}{l}\text { Microtubules } \\
\text { mass }^{c^{\circ}}\end{array}$ & $\begin{array}{l}\text { Microtubule } \\
\text { profile } \\
\text { density }^{d}\end{array}$ \\
\hline Control ( $9 \mathrm{hr})$ & $0.58 \pm 0.06 n=16$ & $480^{c}$ & 127 & 278 & $27 \pm 4.4 n=16$ & 12,960 & 104 \\
\hline 165 nm nocodazole & $1.01 \pm 0.16 n=14$ & $568 \pm 124 n=14$ & 454 & 504 & $43 \pm 3.9 n=25$ & 24,424 & 53 \\
\hline $330 \mathrm{~nm}$ nocodazole & $2.00 \pm 0.16 n=21$ & $270 \pm 49 \quad n=14$ & 848 & 511 & $66 \pm 5.9 n=21$ & 17,820 & 21 \\
\hline
\end{tabular}

"Axon volume was calculated from $v=\pi r^{2} \times l$, where $r=$ radius and $l=$ axon length (total outgrowth from dissociated cells)

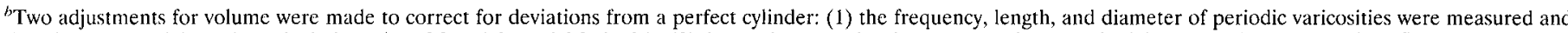

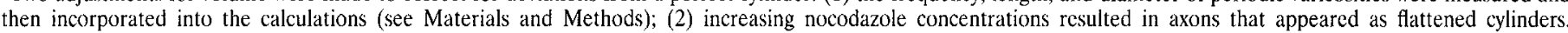
Calculations were also adjusted for the flattening effect by assuming that the axon cross-section in the nocodazole-treated cultures was represented by an oval.

'Microtubule mass was calculated by multiplying axon length (total outgrowth from dissociated neurons) by the average number of microtubules per axon cross-section.

${ }^{d}$ Microtubule profile density refers to the density of cross-sectioned microtubules profiles per axonal cross-sectional area $\left(\mu \mathrm{m}^{2}\right)$.

${ }^{\prime}$ In this case, explant outgrowth from one explant was used instead of measuring the length of neurites from individual neurons.

bules, they more firmly support the hypothesis that the decreased advance rate results from another cause, decreased dynamic instability.

\section{MATERIALS AND METHODS}

Cell culture. The cell culture methods used were the same as those described previously (Bridgman and Dailey, 1989). Briefly, they involved removal of the superior cervical ganglion (SCG) from E20 rat embryos. Ganglia were desheathed and either cut into small pieces for explant cultures or dissociated with trypsin for dissociated cell cultures. All cultures were grown in medium containing $10 \%$ horse serum and chick embryo extract at $37^{\circ} \mathrm{C}$ in a $5 \% \mathrm{CO}_{2}$ atmosphere. Nocodazole was prepared as a $10 \mathrm{mg} / \mathrm{ml}$ stock in DMSO and stored frozen at $-70^{\circ} \mathrm{C}$. For treatment of cultures, the stock was serially diluted in L15 followed by culture medium to the appropriate concentration immediately before use. For long-term cultures, the nocodazole-containing medium was replenished at $24 \mathrm{hr}$ intervals.

Immunofluorescence. Cultured cells were fixed with $0.25 \%$ glutaralde-

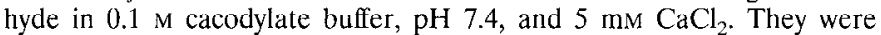
processed for microtubule immunofiuorescence as described previously using a monoclonal antibody to $\beta$-tubulin (East Acres Biological, Southbridge, MA) (Dailey and Bridgman, 1989). Splayed microtubule preparations were prepared as described by Brown et al. (1993). Monoclonal antibodies to tyrosinated $\alpha$-tubulin (YL 1/2, Accurate Chemical, Westbury, NY) were used in double-labeling experiments. The cells were mounted on slides using an anti-bleaching reagent (Vecta-shield, Vector Laboratories, Burlingame, CA) and then photographed with T-Max 400 film or imaged with a slow scan, cooled CCD (Photometrics, Tucson, AZ) on an Olympus inverted microscope with a $63 \times$, N.A. 1.4 or $40 \times$, N.A. 1.0 lens.

Electron microscopy. For thin-section EM, cultures were fixed with glutaraldehyde as above for immunofluorescence. They were then rinsed with buffer without $\mathrm{CaCl}_{2}$, post-fixed with $1 \%$ osmium, stained en block with $1 \%$ uranyl acetate, dehydrated in a graded series of acetone, and embedded in Araldite. Thin sections were cut either as cross-sections or longitudinally, parallel to the substratum.

Measurement of axon length and volume. In explant cultures (plus or minus nocodazole), phase-contrast photographs were taken on three sides of each explant at $25 \times$ optical magnification, a low-magnification dark-field image $(6.25 \times)$ was also taken to aid orientation. When cultures wcrc grown $>24 \mathrm{hr}$, multiple overlapping photographs were nccessary to encompass the entire length of outgrowth. Micrographs were used to measure the distance from the edge of the explant to the main distal growth cone field. The outgrowth for each explant was determined from the average of the three measurements.

Dissociated neurons grown in low-density cell culture (plus or minus nocodazole) were used to determine the total length of outgrowth. Cells producing axons that were not intermingled with axons of neighboring cells were photographed using phase-contrast microscopy $(25 \times$ optical magnification). All processes and their branches were traced on micrographs, their lengths were measured, and then the total was summed for each cell. Because dissociated cell axons gencrally grew straight, no attempt was made to compensate for any slight curvature of axons.
Axon volume measurement was carried out by multiplying the length of the axon outgrowth by the average cross-sectional area of axons as determined from electron micrographs (see legend to Table 1). This value was adjusted to take into account the presence of varicosities. We measured the number of varicosities along the length of axon of dissociated cells of control and nocodazole-treated cultures observed by phasecontrast microscopy. For control cultures, there were 51 varicosities along the $1024 \mu \mathrm{m}$ of total length measurcd. For the $165 \mathrm{~nm}$ nocodazole-treated cells, there were 14 varicosities along the $568 \mu \mathrm{m}$ of axon measured. For the $330 \mathrm{~nm}$ nocodazole-treated cclls, no varicosities were detected. For the control and $165 \mathrm{nM}$-treated cells, we then calculated the increase in axon cylinder volume resulting from varicosities by using the average maximum diameter and an adjusted length of the varicosities measured from longitudinally cut axons in EM micrographs. An adjusted varicosity length was used because the maximum diameter was only reached at the center of the varicosity, i.e., the ends of the varicosity taper. The adjusted length was derived cmpirically from arca mcasurcments of several longitudinally cut varicosities and was approximately equal to one-half of the actual average varicosity length. There was no detectable difference in varicosity length and maximum diameter between control and $165 \mathrm{~nm}$ nocodazole-treated samples; average varicosity maximum diameter was $2.5 \mu \mathrm{m}$, and the adjusted length averaged $1.5 \mu \mathrm{m}$.

Measurement of lengths of fluorescently labeled microtubule segments. The lengths of segments of microtubules labeled intensely with antityrosinated tubulin were determined on preparations subjected to the splaying protocol (see above). This was not difficult in the case of axonal microtubules because of the relatively short segments that were brightly labeled. Microtubules near the growth cone tended to have long segments that were brightly labeled, making identification of the proximal extent of this staining difficult. However, the splayed preparations were variable in the degree of splaying and, in particularly favorable preparations, it was possible to trace many of the intensely labeled segments from their plus ends to the proximal end of the staining. Measurements were carried out on a Sun Workstation graphics monitor. Images were rendered such that $5 \mu \mathrm{m}$ corresponded to $-1 \mathrm{~cm}$ on the screen, and a ruler was held up to the screen to measure the lengths of individual microtubule segments.

\section{RESULTS}

\section{Nocodazole alters axon outgrowth and axon morphology}

We first established the dose-response characteristics of SCG explant outgrowth to nocodazole (Fig. 1, inset). Concentrations of 165 and $330 \mathrm{~nm}$ had an approximately half-maximal effect and were used for subsequent measurements. Because the outgrowth from treated explants appeared to be more sparse than that from control explants, we investigated the possibility that nocodazole inhibits axon initiation, rather than axon extension rate. Measurements of explants taken at multiple time points indicated that explant outgrowth rate was decreased (Fig. 1). These measurements were carried out over a $4 \mathrm{~d}$ period, during which outgrowth 


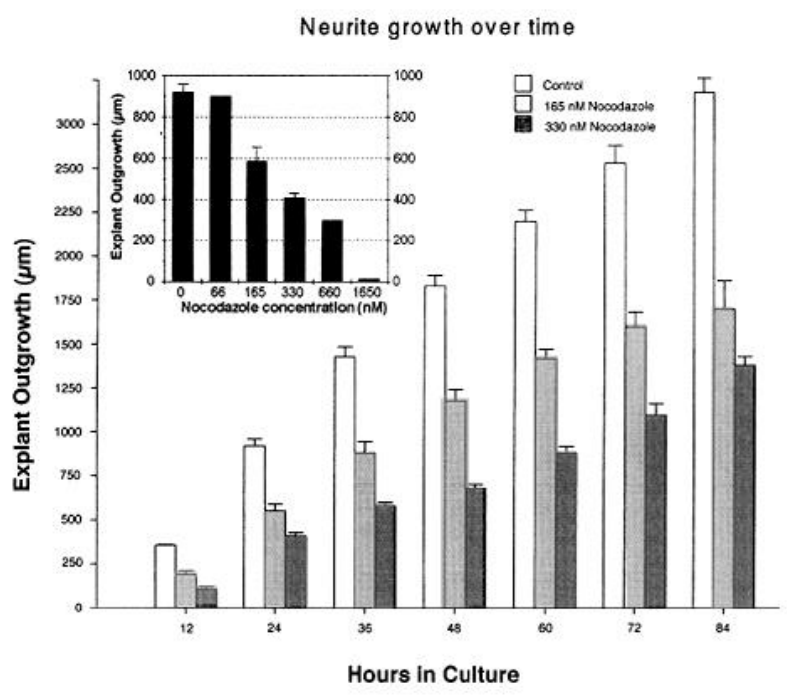

Figure 1. Inset, The effect of different concentrations of nocodazole on the radial outgrowth from explants grown for $24 \mathrm{hr}$. Except for the 1650 $\mathrm{nM}$ concentration, bars without error bars indicate measurements on a single explant as described in Materials and Methods. At the $1650 \mathrm{~nm}$ concentration, the SEM was too small to indicate on the graph. Error bars indicate SEM. Low concentrations of nocodazole influence the radial outgrowth from explants over an extended period of time. At the indicated times, phase-contrast micrographs were taken of live explants. Measurements were taken from these photographs as described in Materials and Methods. Error bars indicate SEM.

continued. This suggests that nocodazole is less toxic than vinblastine, which was lethal to neurons within $3 \mathrm{~d}$ at half-maximal concentrations for outgrowth inhibition (Baas and Ahmad, 1993). We also directly examined the effect of nocodazole on neuritogenesis by using dissociated neurons. Twelve hours after plating, $83 \%$ of the control neurons had axons, but only 71 and $46 \%$ of the 165 and $330 \mathrm{~nm}$ nocodazole-treated neurons had axons, respectively. Inhibition of neuritogenesis was reversed (qualitatively) by washout, suggesting that this effect was not attributable to cell death. Thus, nocodazole prevents neuritogenesis in a subpopulation of axons, and axons that do extend do so at a slower rate. Our subsequent efforts were aimed at understanding the mechanism by which nocodazole slowed the rate of axon extension.

Two morphological changes in axons were observed that may be relevant to the mechanism of the action of nocodazole on axon extension. Both axon diameter and growth cone size appeared to have increased. This effect was only seen if cells were directly plated and grown in nocodazole-containing medium. If cells were first grown in normal medium and then transferred to nocodazolecontaining medium, no increase in size was seen for several hours after treatment. The first possibility that we wished to examine was that the increase in axon diameter combined with the slower advance rate indicated that nocodazole had no effect on the rate of axon assembly. To evaluate this possibility, transverse sections of axons emanating from explants were processed for electron microscopic observation, and cross-sectional areas were determined (Fig. 2, Table 1). The average volume of axoplasm per neuron was estimated by multiplying the average axon crosssectional area of axons by the radius of explant outgrowth or the average of the total length of axon produced by individual dissociated neurons. A nonsignificant increase in volume $(\sim 10 \%)$ was observed for the nocodazole-treated explants. The changes in axon diameter and growth cone size are likely to be secondary to the reduction of axon lengthening rate (see Discussion).

\section{Nocodazole does not alter microtubule production}

Given that nocodazole was not affecting the rate of axoplasm production, we determined whether the net rate of axonal microtubule production was affected. Axonal microtubule mass was estimated by counting the number of microtubule profiles in electron micrographs of axon sections and multiplying by the average total length of axon per neuron as for the volume calculation. An increase in the number of microtubules/cross-section in nocodazole-treated axons compensated for the decrease in average total axon length (Table 1). We found no difference in microtubule mass produced per unit time for $165 \mathrm{~nm}$ nocodazole, and only a $20 \%$ decrease in microtubule mass for 330 nм nocodazole (despite a $380 \%$ decrease in axon extension rate). This finding and our observation of continuous outgrowth over a $4 \mathrm{~d}$ period in the presence of nocodazole suggest that nocodazole does not inhibit microtubule formation. It is possible, however, that the axonal microtubule mass is derived from short microtubules present in the cells before the nocodazole treatment, and that nocodazole, like vinblastine, does inhibit microtubule formation. To test this possibility, we determined whether low doses of nocodazole would prevent microtubule formation if microtubules are disassembled before exposure to the low doses of nocodazole.

To cause complete microtubule disassembly before plating, explants were pretreated for $6 \mathrm{hr}$ with $33 \mu \mathrm{M}$ nocodazole, a dose that had been shown previously to depolymerize $\sim 95 \%$ of the microtubule mass in SCG neurons and prevent microtubule production (Baas and Ahmad, 1992). To check the effectiveness of this treatment, we prepared cells that had been subjected to this treatment for EM. Thin sections were cut through explants, and multiple sections from 71 neuronal cell bodies were analyzed. No microtubule profiles were detected at MTOCs or elsewhere in any of the cell bodies examined (data not shown). Two processes that interdigitated between cell bodies did show several microtubule profiles (data not shown). These profiles were associated with intermediate filaments, suggesting that such an association protects microtubules from disassembly. Nonetheless, our data corroborate previous findings (Baas and Ahmad, 1992) and support the view that pretreatment with a high dose of nocodazole eliminates the putative store of microtubules. Despite the extensive disassembly caused by the pretreatment, explants plated in media containing no nocodazole or either of the low doses of nocodazole exhibited outgrowth rates that were the same as those observed in nonpretreated explants (compare data in Table 2 with those in Fig. 1). The density of the outgrowth was also unaffected by the pretreatment. Furthermore, electron microscopic observations directly demonstrated that microtubules were again present at MTOCs. Thus, nocodazole treatment increased axon diameter and the number of microtubules per cross-section, but did not affect the rate of production of axoplasm or microtubules.

Although we suspected that the increase in microtubules per cross-section described above was a consequence of the effects of nocodazole on microtubules, it was formally possible that it resulted from an indirect effect of nocodazole on the rate of axon lengthening. To test this, we measured axon diameter and microtubules per cross-section in untreated axons that were as short as the nocodazole-treated axons (grown for $9 \mathrm{hr}$ rather than $24 \mathrm{hr}$ ). Short, untreated axons were slightly thicker and higher in microtubules per cross-section than longer control axons, but these values were significantly lower than those of nocodazole-treated neurons (Table 1). This illustrates that the reduced axon length caused by nocodazole does not produce the increase in microtu- 

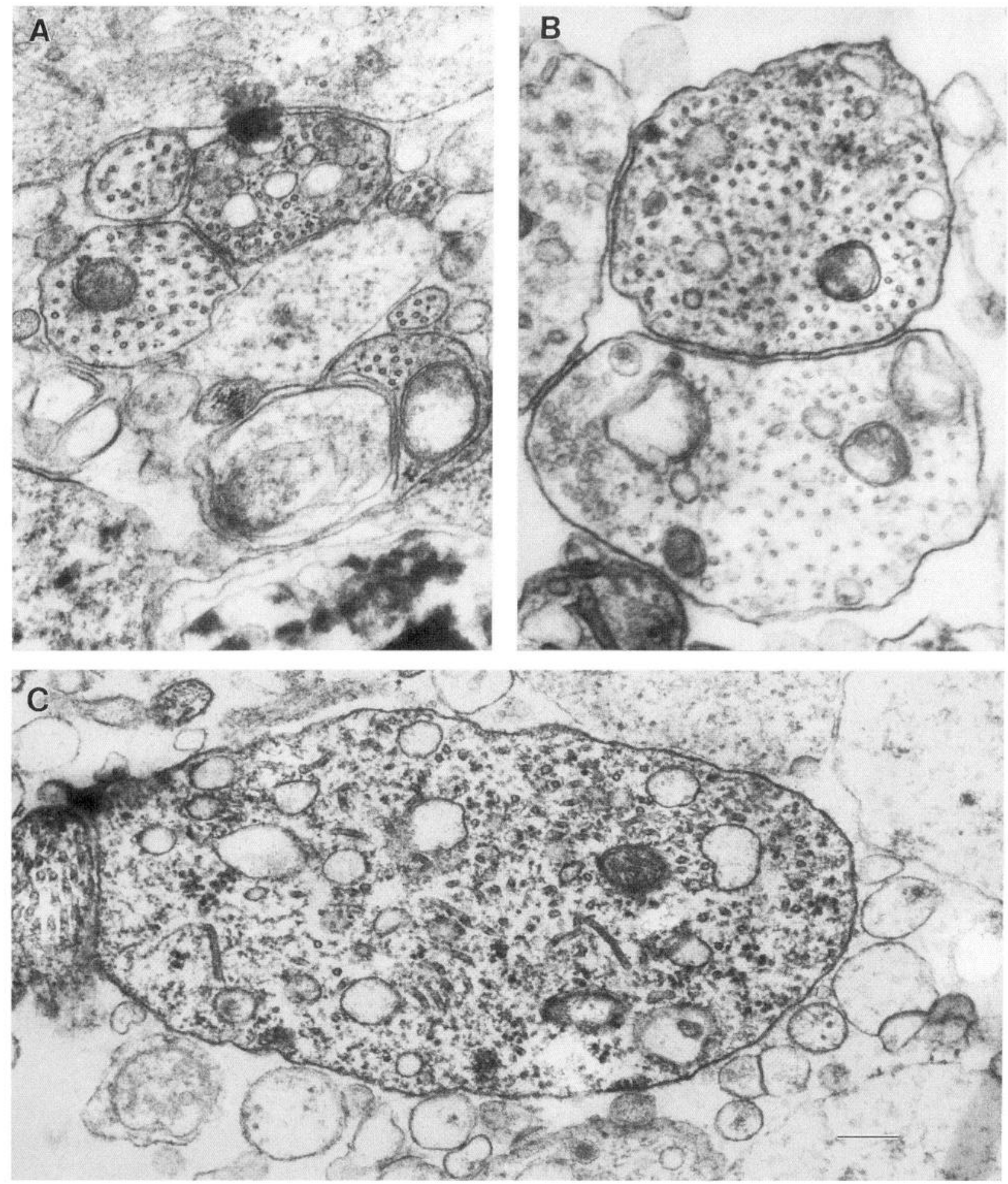

Figure 2. The effects of low concentrations of nocodazole on axon diameter and the number of microtubules per axon cross-section. Cultures were grown for $16 \mathrm{hr}$. All sections were taken just as the axons exited the explants (determined by observing multiple thick sections by light microscopy just before cutting thin sections). $A$, Cross-section from a control culture showing several axons. $B$, Cross-section from a culture plated in 165 nm nocodazole. Two axons are shown. The axons are larger in diameter and contain greater numbers of microtubules than those from control cultures. $C$, Cross-section from a culture plated in 330 nM nocodazole. A single axon is shown. The axon has a larger cross-sectional area and greater numbers of microtubules than controls or $165 \mathrm{~nm}$-treated cultures. Note the oval shape of the axon and less organized microtubule array. Semiserial sectioning revealed that the oval shape is not a result of oblique sectioning and that the microtubules running at oblique angles are an unusual property of these treated axons. Scale bar, $0.2 \mu \mathrm{m}$.

bules per cross-section. The morphological changes that we observed may be secondary to the reduction in the rate of axon lengthening. A decrease in lengthening rate without a corresponding decrease in rate of axoplasm production would be expected to bring about an increase in axon diameter and, therefore, growth cone size. We noted that microtubule density, i.e., microtubule profiles/unit cross-sectional area, decreased with increasing volume, consistent with the possibility that axon caliber and micro- 
Table 2. Axon and microtubule lengths

\begin{tabular}{|c|c|c|c|c|}
\hline $\begin{array}{l}\text { Nocodazole } \\
\text { concentration }\end{array}$ & $\begin{array}{l}\text { Radial outgrowth length }(\mu \mathrm{m}) \\
\text { after pretreatment with } 33 \mu \mathrm{M} \\
\text { nocodazole }^{a}( \pm \text { SEM }) n=3\end{array}$ & $\begin{array}{l}\text { Average microtubule length } \\
\text { index }(\mu \mathrm{m})( \pm \mathrm{SEM})^{b}\end{array}$ & $\begin{array}{l}\text { Length of bright tyrosinated } \\
\text { tubulin staining in axon } \\
\text { shaft }(\mu \mathrm{m})( \pm \mathrm{SEM})\end{array}$ & $\begin{array}{l}\text { Length of bright tyrosinated } \\
\text { tubulin staining in growth } \\
\text { cone }(\mu \mathrm{m})( \pm \mathrm{SEM})\end{array}$ \\
\hline 0 & $825 \pm 14$ & $204 \pm 9$ & $12 \pm 1.1^{c}(6 \%), n=23$ & $81.9 \pm 6.4^{d}(40 \%), n=10$ \\
\hline $165 \mathrm{nM}$ & $600 \pm 40$ & $260 \pm 21$ & Not determined & Not determined \\
\hline $330 \mathrm{nM}$ & $567 \pm 23$ & $256 \pm 13$ & $4.5 \pm 0.19^{c}(2 \%), n=39$ & $54.3 \pm 1.7^{d}(21 \%), n=12$ \\
\hline
\end{tabular}

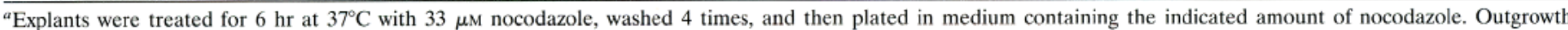
measurements were made $24 \mathrm{hr}$ after plating; compare to lengths in Figure 1.

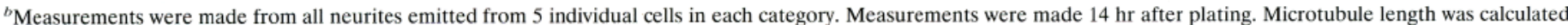

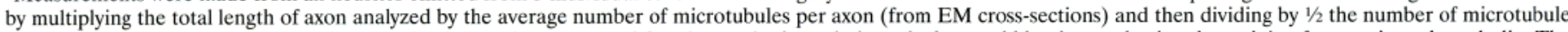

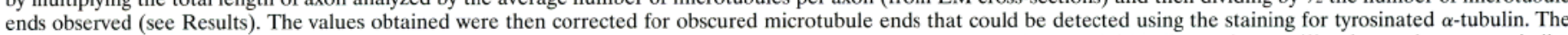

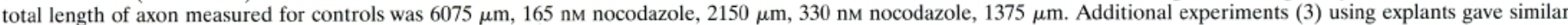
results, although the values calculated for microtubule length were larger.

${ }^{c}$ Difference between means was significant ( $t$ test; $p<0.001$ ).

${ }^{d}$ Difference between means was significant ( $t$ test; $p<0.005$ ).

tubule density are independently determined (see Discussion). We next investigated whether nocodazole affects the average lengths of microtubules.

\section{Nocodazole does not alter average microtubule length}

Measuring microtubule lengths in axons is complicated by the fact that microtubules are long, averaging $100 \mu \mathrm{m}$ in previous studies (Bray and Bunge, 1981), and densely packed. Thus, it is not feasible, under normal culture conditions, to follow many individual microtubules from plus end to minus end in intact axons, and indirect methods must be used. We therefore combined the microtubule splaying technique introduced by Brown et al. (1993) with a modification of a microtubule length measurement strategy developed by Bray and Bunge (1981). Average microtubule length is equal to the total length of microtubules in a set of axons divided by the number of microtubules. The total length of microtubules is equal to the product of the average number of microtubules per cross-section and the total length of axons. To estimate the number of microtubules in axons, we counted the number of ends that were detected after debundling and splaying of microtubule ends (see Materials and Methods; see also Brown et al., 1993) (Fig. 3). Half of this number is the number of detected microtubules. A number of factors interfered with the detection of ends. Although we took steps to minimize these influences (discussed next), it is unlikely that we eliminated them altogether. Therefore, we view the value we obtained for the summed microtubule lengths divided by the number of microtubules as an informative overestimate of microtubule length, and refer to this value as a microtubule length index.

The principle factor limiting the detection of microtubule ends is that the splaying is not adequate to resolve all of the ends; some ends overlap with other microtubules and are not counted. To minimize this, we stained splayed preparations with both anti- $\beta$ tubulin and anti-tyrosinated $\alpha$-tubulin (Fig. 3). Tyrosinated tubulin is concentrated only at the plus ends of microtubules in axons (Baas and Black, 1990; Brown et al., 1993). Therefore, overlap of plus ends and minus ends could be detected. We assumed that an equal proportion of minus ends was obscured by overlap, and we adjusted our estimate of the microtubules per unit length accord-
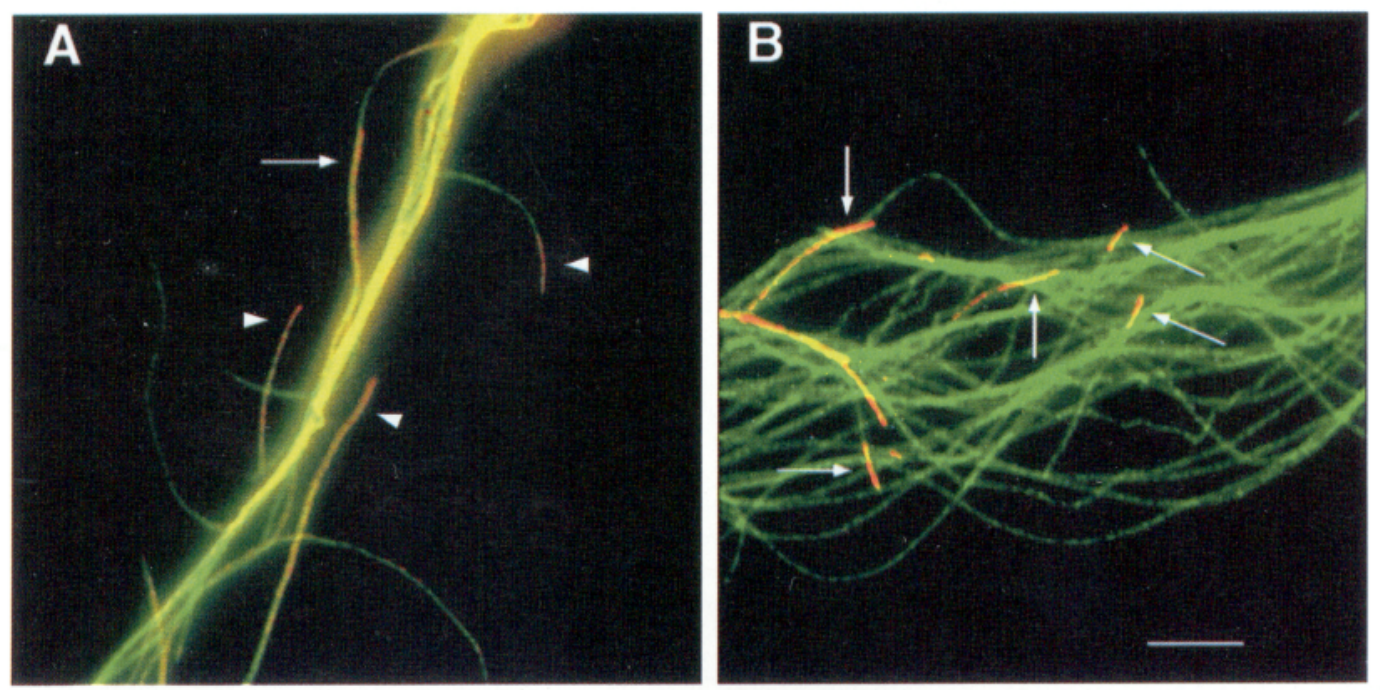

Figure 3. Labeling of splayed microtubule preparations for $\beta$-tubulin (green) and tyrosinated $\alpha$-tubulin (red) reveals endings obscured by overlap with detyrosinated $\alpha$-tubulin. $A$, Control neurite; $B, 165 \mathrm{~nm}$ nocodazole-treated neurites. Tyrosinated $\alpha$-tubulin staining labels the plus ends of microtubules within control $(A)$ and nocodazole-treated $(B)$ neurites (arrows and arrowheads). This staining reveals microtubule endings that may otherwise have escaped detection (arrowheads). Although the microtubules in the nocodazole-treated neurite do not splay out as far from the neurite as in the control neurites, the shorter lengths of tyrosinated microtubule segments in the nocodazole-treated neurites result in less overlap of tyrosinated endings. Scale bar, $11 \mu \mathrm{m}$. 
ingly. We found that $35-45 \%$ of the endings had not been detected with the $\beta$-tubulin staining alone. After adjusting for these uncounted ends, the value that we obtained for average microtubule length in control axons $(200 \mu \mathrm{m}$, Table 2$)$ was still about twice that reported by others. This is caused in part by overestimation and may also be caused by differences in cell types used in other systems. Even though our index is an overestimation of microtubule length, our comparison between control and experimental axons is still valid. Although we cannot be certain that the same percentage of ends was detected for both treated and untreated axons, endings seemed to be more easily resolved in nocodazole-treated controls because of shorter lengths of tyrosinated $\alpha$-tubulin staining (Fig. 3; see below). This would tend to decrease the estimation of microtubule length in the nocodazoletreated samples compared with the controls. Thus, because no significant differences in average microtubule length indices were found between treated and control axons, it is unlikely that nocodazole caused a decrease in microtubule length.

\section{Nocodazole may decrease the dynamic instability of microtubules in growth cones and axons}

Nocodazole decreases the dynamic instability of microtubules in vitro (Jordan et al., 1992). A decrease in dynamic instability should have at least two effects on microtubules: (1) a decrease in the variability of microtubule lengths, and (2) a decrease in the turnover of tubulin in microtubules. Fewer catastrophic events will occur, increasing the persistence of stable portions of microtubules, and fewer rapid polymerization events will occur, decreasing the percentage of microtubule length that is newly assembled at any given time. In other words, the proportion of the microtubule (plus end) that is kinetically active will be shorter. Direct measurement of microtubule dynamics in growth cones is complicated by the bundling of microtubules in axons close to their tips. Inability to identify long stretches of microtubules makes it difficult to distinguish polymerization kinetics from sliding (Tanaka et al., 1995). Nonetheless, we made two observations that suggest that dynamic instability of microtubules is decreased in nocodazole-treated neurons.

The distribution of microtubule endings in the growth cone suggested that the dynamic properties of microtubules were blunted by nocodazole. Normally, microtubules in the growth cone splay apart, often bend sinuously, and penetrate different distances into the growth cone periphery before ending (Fig. 4A). This diversity of lengths and the highly curved paths presumably result from the variability of polymerization/depolymerization events associated with normal dynamic instability of microtubules and perhaps sliding. In nocodazole-treated cultures, microtubule endings were more compacted together, did not bend sinuously, and did not penetrate into the P-domain (Fig. 4B,C). Indeed, microtubules in treated growth cones ended at about the same axial location in the $\mathrm{C}$-domain, splaying out slightly to give a brush-like appearance (Fig. $4 B, C$ ). Acute application of nocodazole $(165$ or $330 \mathrm{~nm}$ ) induced this pattern in $\sim 50 \%$ of the growth cones within $30 \mathrm{~min}$, and this pattern was maintained for up to 108 hr during continuous nocodazole treatment (data not shown). The effect was reversed within $30 \mathrm{~min}$ of washout (data not shown). The uniform appearance of the normally dynamic microtubule plus ends in nocodazole-treated growth cones is consistent with the possibility that nocodazole reduces dynamic instability of microtubules. We next investigated whether low doses of nocodazole affected the distribution of tyrosinated or acetylated microtubules in neurons.
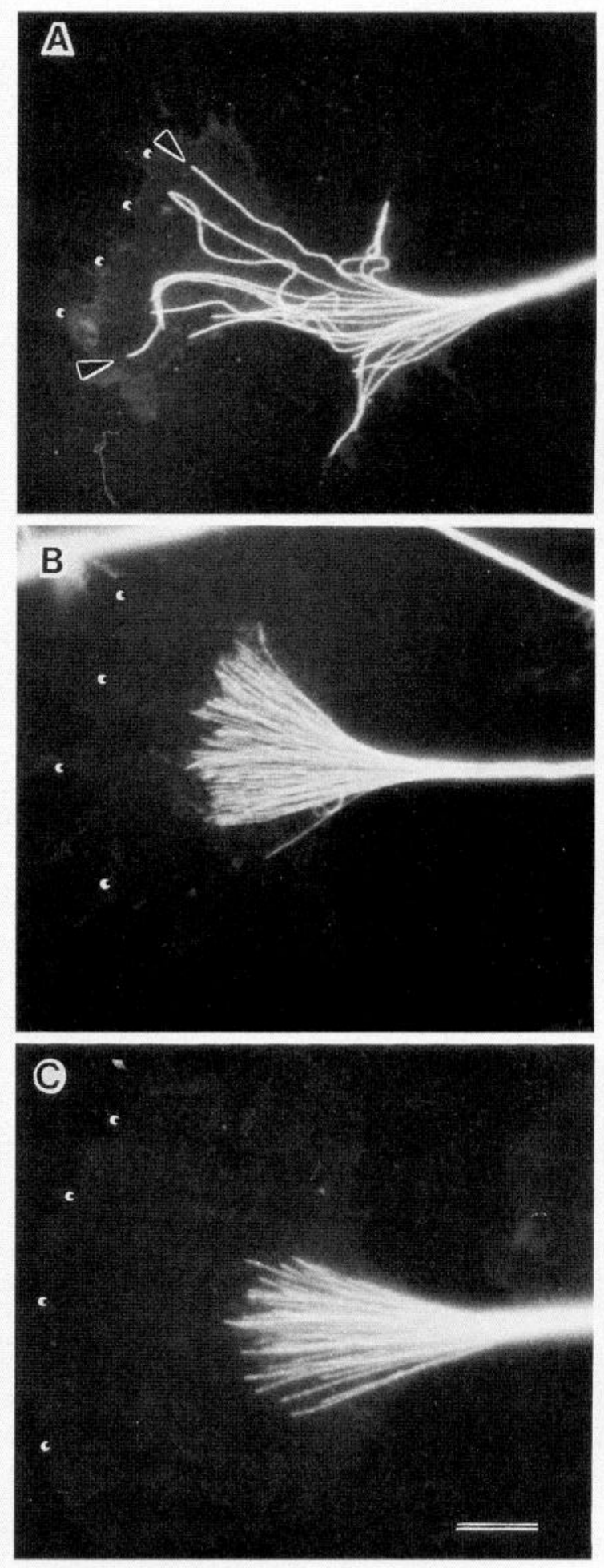

Figure 4. The effects of low concentrations of nocodazole on the distribution of microtubules (labeled with an antibody to $\beta$-tubulin) in growth cones. $A$, A growth cone from a control culture. Note that microtubules sometimes penetrate out into the peripheral domain of the growth cone (arrowheads). B, A growth cone from a culture grown in 165 nm nocodazole. The numbers of microtubules ending in the growth cone appear increased compared with control. Microtubules are restricted to the central domain, and their endings are closely spaced. $C$, A growth cone from a culture grown in $330 \mathrm{~nm}$ nocodazole. The distribution of microtubules is similar to that seen in $B$. Note that the axonal microtubule bundle width is also increased. The dots indicate the boundary of the leading edge. Scale bar, $9 \mu \mathrm{m}$. 
Detyrosination and acetylation of $\alpha$-tubulin increase with the time during which the tubulin has been incorporated into polymer, i.e., the age of the microtubulc scgment (Baas and Black, 1990; Baas et al., 1991; Brown et al., 1993). Acetylation has also been shown to correlate with increased microtubule stability in neurons (Lim et al., 1989; Li and Black, 1996). We stained ncurons with antibodies that recognize exclusively tyrosinated or acetylated $\alpha$-tubulin to determine whether the relative distributions of newly or previously polymerized tubulin are altered by nocodazole treatment. Because of the large difference in the brightness values between the bundled microtubules in axons and the individual microtubules in growth cones, we rendered microtubule staining in pseudocolor (Fig. $5 A, B, D, E$ ). Because pseudocolor maps brightness to a color, the microtubule staining in the growth cone can be observed without causing the axon staining to saturate. Minimizing saturation was necessary for comparing the relative distributions of tyrosinated and acetylated microtubules in both growth cones and axons. For purposes of comparing the relative distributions of the two staining patterns to each other, the brightness of the raw image was adjusted so that $-1 \%$ of the pixels had brightness values greater than the peak brightness, i.e., $1 \%$ of the pixels were saturated. Color overlays of the microtubule staining are also provided for comparison of the staining distributions of the two antigens (Fig. 5C,F). Please note an unintended consequence of pseudocoloring: fluorescent "flare" from particularly bright areas to adjacent pixels was sometimes bright enough to be mapped to a color different from the background, giving the false impression of increased width [e.g., the proximal neurite segment $(* *)$ in Fig. $5 B$ appears wider than in Fig. $5 A, C]$.

Microtubules in untreated growth cones (Fig. $5 A$, arrows, arrowhead) and distal portions of axons (Fig. $5 A$, *) stained brightly for tyrosinated tubulin (cf. Gordon-Weeks and Lang, 1988). The distal portions of axons (Fig. $5 A,{ }^{*}$ ) were more brightly stained than proximal portions (Fig. $5 A, * *$ ). The distribution of acetylated tubulin was the complement of tyrosinated tubulin, being more intense in proximal axon segments (Fig. $5 B$, ***) than in distal axon segments (Fig. $5 B,{ }^{*}$ ) and growth cones (Fig. $5 B$, arrows, arrowhead) (cf. Lim et al., 1989). In most cases, acetylated microtubule staining did not extend to the distal tips of microtubules in the growth cone (Fig. 5A,B, arrows), but exceptions were found (arrowheads). Nocodazole treatment did not change the overall staining pattern of tyrosinated tubulin (but see bclow); it remained brighter in the growth cone and distal axon (Fig. 5D, left panel) than in the proximal axon (Fig. $5 D$, right panel). However, nocodazole did alter the distribution of acetylated $\alpha$-tubulin, increasing the brightness of acetylated tubulin staining in the growth cone and distal axon (Fig. $5 E$, left) so that it was comparable with that in proximal axon segments (Fig. $5 E$, right). Note that the increase in neurite breadth near the growth cone does not seem to correspond to an increase in neurite thickness. Staining for $\beta$-tubulin revealed that this broadening corresponds to a decrease in tubulin staining intensity, suggesting looser packing of microtubules and a decrease in neurite thickness (data not shown). As in untreated growth cones, the tyrosinated tubulin staining extended out to the tips of microtubules (Fig. 5D, region indicated by arrow), whereas acetylated tubulin staining became attenuated, only rarely persisting in brightness to the tips (Fig. $5 E$, arrowhead). These data suggest that microtubule segments in growth cones and distal axons are older and, therefore, likely to be more stable than microtubules in untreated growth cones.

We frequently observed the presence of one or a few microtubules in nocodazole-treated growth cones that were almost exclu- sively stained by the antibody that recognizes acetylated $\alpha$-tubulin (Fig. 5D,E, open arrowheads). These microtubules were typically highly curved or bent (cf. Gundersen and Bulinsky, 1984). Their sinuosity and staining profile are consistent with the possibility that they moved, already polymerized, from the axon into the growth cone. Forward movement of microtubules within untreated (Tanaka and Kirshner, 1991) and vinblastine-treated growth cones (Tanaka et al., 1995) has been described in amphibian neurons.

We extended our analysis by comparing the lengths of tyrosinated $\alpha$-tubulin-rich (newly assembled) microtubule segments between nocodazole-treated and control microtubules in dissociated SCG neurons. In both control and nocodazole-treated neurons, the most intense tyrosinated $\alpha$-tubulin staining was found near the growth cone (see above), and the second most intense staining was found near the cell body (data not shown). Highermagnification images of splayed preparations showed that microtubules in between the proximal and distal regions of the axon contained short lengths of tyrosinated $\alpha$-tubulin at their plus ends (Fig. 6). At the growth cone, the lengths of microtubule segments labeled with tyrosinated $\alpha$-tubulin are much greater than those in intermediate regions of the axon (compare Fig. 6 with Fig. 5; note difference in magnification). We measured the lengths of microtubules enriched in tyrosinated $\alpha$-tubulin in the axon (excluding near the soma) and at the growth cone. The axon segments adjacent to the soma were excluded because the staining of tyrosinated $\alpha$-tubulin usually had its proximal terminus in the cell body, making it impossible to identify. In both the growth cone and the more proximal axonal segments, the length of the tyrosinated $\alpha$-tubulin staining was significantly longer in control preparations than in nocodazole-treated preparations (Fig. 6, Table 2). Given that the average length of microtubules is not altered by nocodazole treatment, these data indicate that the percentage of microtubule length that is composed primarily of tyrosinated $\alpha$-tubulin is decreased by nocodazole. As expected, we also found that the proportion of microtubule length brightly stained for acetylated tubulin was roughly complementary to that of the tyrosinated $\alpha$-tubulin staining (Fig. 6, most clearly in $B$ ). The shift in the percentage of microtubule length consisting primarily of tyrosinated or acetylated tubulin implies that nocodazole decreases gross plus end polymerization relative to the detyrosination and acetylation processes, and is consistent with the possibility that dynamic instability is decreased by nocodazole.

\section{DISCUSSION}

To understand better the contribution of microtubule dynamics to axon outgrowth, the effects of chronic treatment with low concentrations of nocodazole on axon outgrowth and microtubule organization in SCG neurons were examined. Several features of axon assembly were affected by nocodazole: extension rate, axon diameter, microtubule profiles per cross-section, the distribution of microtubule endings at the growth cone, and the ratio of tyrosinated to detyrosinated or acetylated microtubule lengths. Of equal interest are the axon assembly properties that were not altcred by nocodazole treatment: rate of production of axonal volume (i.e., axoplasm production), net rate of microtubule production, and length of microtubules. As will be discussed, these data are consistent with the possibility that dynamic instability of microtubules has a critical role in modulating axon assembly.

Our study contributes significantly to the elegant work recently published by Tanaka et al. (1995), which reached a similar conclusion based on changes in growth cone advance and in the 

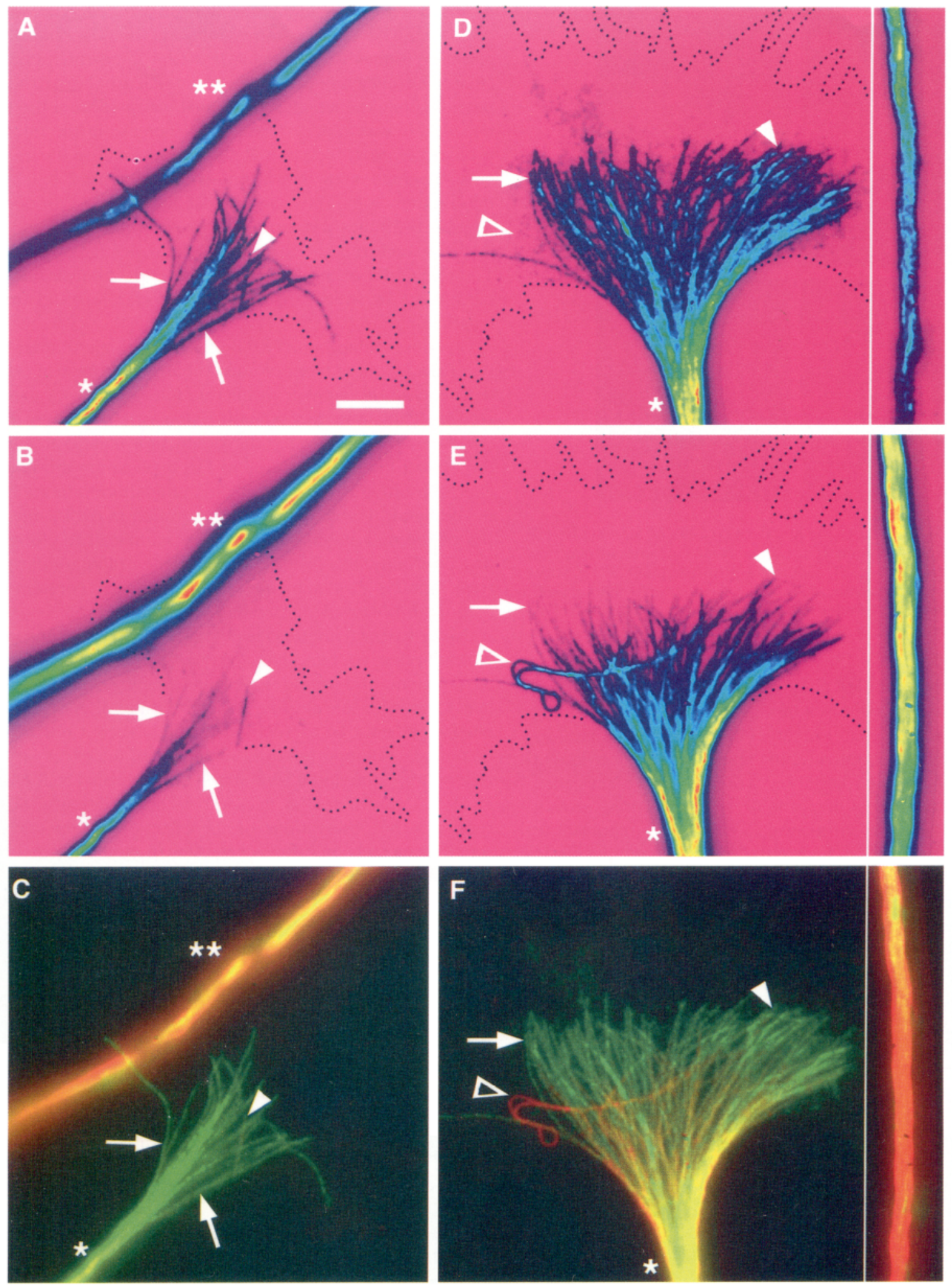

Figure 5. The effect of nocodazole treatment on the distributions of tyrosinated $\alpha$-tubulin and acetylated $\alpha$-tubulin in growth cones and neurites. $A$ - $C$, Untreated growth cone and neurite. $D-F, 330 \mathrm{~nm}$ nocodazole-treated growth cone (left) and a segment of its neurite (right) centered $110 \mu \mathrm{m}$ proximal to the growth cone. $A, B$, Pseudocolor renderings of anti-tyrosinated $\alpha$-tubulin staining ( $A$, fluorescein) and anti-acetylated $\alpha$-tubulin staining ( $B$, Cy3). $C$, Color overlay of $A$ (green) and $B($ red $)$. In the growth cone, the tyrosinated tubulin stains out to the tips of the microtubules $(A)$. Acetylated tubulin staining is faint but detectable in the growth cone $(B$, arrows) and occasionally labels out to the tips brightly $(B$, arrowhead). By comparing the neurite for which the growth cone has advanced out the field $\left({ }^{* *}\right)$ with the distal neurite segment $\left({ }^{*}\right)$, it is evident that tyrosinated tubulin staining is brighter distally than proximally, and the converse is true for the acetylated tubulin. This is also indicated in $C$, in which the tyrosinated tubulin staining dominates the growth cone and distal neurite, but the acetylated tubulin staining dominates the proximal neurite. $D, E$, Pseudocolor renderings of anti-tyrosinated $\alpha$-tubulin staining $(D$, fluorescein) and anti-acetylated $\alpha$-tubulin staining $(E, C y 3) . F$, Color overlay of $A$ (green) and $B($ red $)$. As for the untreated growth 

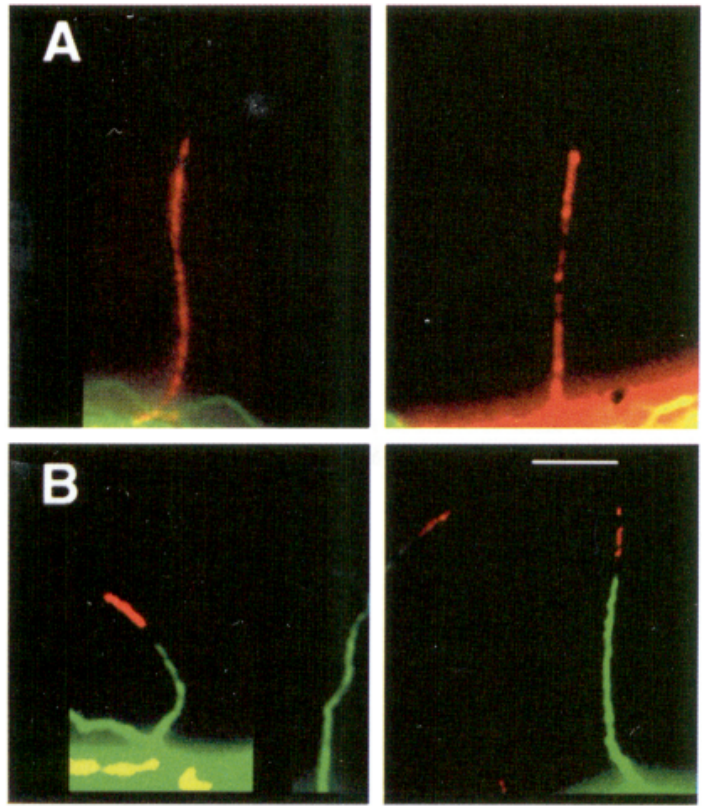

Figure 6. Nocodazole decreases the length of tyrosinated staining found at the plus ends of microtubules splayed out from segments of neurites proximal to the growth cone. $A$, Control neurite segment. $B, 165 \mathrm{~nm}$ nocodazole-treated neurite segment. Note that tyrosinated $\alpha$-tubulin (red, arrowheads) and acetylated tubulin (green) have complementary distributions in microtubules. Scale bar, $2.2 \mu \mathrm{m}$.

distribution of microtubules after acute treatment of Xenopus neural tube cells with low doses of vinblastine. We more firmly establish kinetic variability as the target of our drug treatment by controlling for changes in microtubule length and number and in axoplasm production. Furthermore, Xenopus and mammalian neurons appear to differ in microtubule behavior during growth cone advance (Okabe and Hirokawa, 1992), so it is important to investigate such properties in both systems. By plating our cells in low doses of nocodazole and treating with the drug continuously, we show that the acute effects persist indefinitely. This indicates that dynamic instability is essential to normal growth cone advance. This protocol also led to our observation that neuritogenesis is slowed by low doses of nocodazole. Finally, our findings on the distribution of tyrosinated and acetylated microtubule segments support our conclusion and complement the results of Tanaka et al. (1995).

Low concentrations of nocodazole decrease the rate of axon extension in SCG neurons but do not decrease the rate of axoplasm production. Two observations suggest that this combination of effects caused the increase in axon diameter and growth cone size. First, despite immediate (within $30 \mathrm{~min}$ ) effects on the microtubule array in the growth cone, and a decrease in outgrowth rate, growth cone size and axon diameter do not increase for several hours if nocodazole is added to cultures that were grown initially in the absence of nocodazole. A delay would not be expected if nocodazole directly affected axon diameter or growth cone size. The delay is consistent with the possibility that axoplasm continues to be produced at the same rate, and the decreased advance rate causes the additional axoplasm to be distributed all along the length of axon that was present at the time the nocodazole was added. Second, the increase in the microtubule profiles per cross-section was not proportional to the increase in cross-sectional area, consistent with differential regulation of these parameters. Thus, we propose that because axoplasm production continues unchecked in the presence of nocodazole, a thicker axon forms and this in turn produces a larger growth cone. The remainder of the Discussion, therefore, concentrates on the mechanism by which low doses of nocodazole could slow axon lengthening.

Low doses of nocodazole did not alter net microtubule production (length and number of microtubules were unchanged), but it did increase the number of microtubule profiles per axon crosssection. Thus, nocodazole, unlike vinblastine, does not decrease lengthening by decreasing the production of microtubules. The three most likely alternatives to this explanation are nonspecific effects resulting in poor cell health, an alteration in the bundling or sliding of microtubules, or an alteration in the kinetic properties of the microtubules. Although it is difficult to eliminate the first possibility, the absence of detectable cell death during longterm cultures ( $>4 \mathrm{~d}$ in nocodazole) and the constant rate of outgrowth observed during these treatments argue against nocodazole having a nonspecific deleterious effect on neuron health. No changes in the bundling of axons were apparent from our EM observations, and we did not evaluate sliding. There is no precedent for nocodazole altering those properties of microtubules, but more work must be carried out to assess such a possibility. The final model is supported by observations of the effects of nocodazole on microtubules in vitro and in non-neuronal cells (Jordan et al., 1992), by changes in microtubule organization in SCG neurons, and by observations of the effects of other microtubule stabilizing agents on process extension (Letourneau and Ressler, 1984). This evidence is discussed below.

In addition to its well known effect of inhibiting microtubule polymerization, nocodazole has also been shown to reduce the dynamic instability microtubules in vitro (Jordan et al., 1992). In other words, rapid prolonged polymerization and catastrophic shortening are both suppressed in the presence of nocodazole. Thus, once a microtubule reaches a steady state length based on the free tubulin concentration and the presence of MAPs (Pryer et al., 1992), nocodazole reduces the standard deviation of its length over time, resulting in a more stable microtubule plus end. Two lines of evidence support the presence of such an effect on microtubules in SCG axons. First, the proportion of detyrosinated tubulin in nocodazole-treated axons is greater than in controls. Detyrosination is correlated with stabilization of microtubules in axons (Baas and Black, 1990; Baas et al., 1991; Brown et al., 1993). Second, the distal (plus) ends of microtubules in nocodazoletreated neurons were usually restricted to the base of the growth

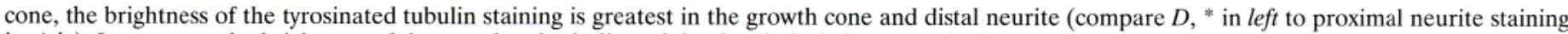

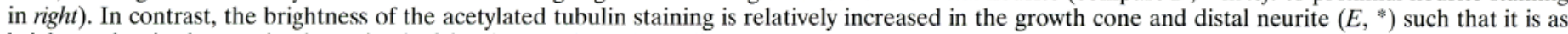

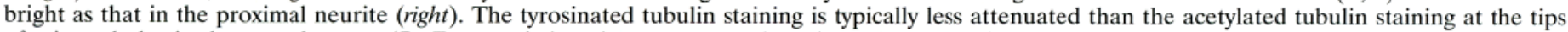

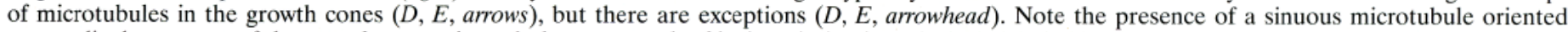

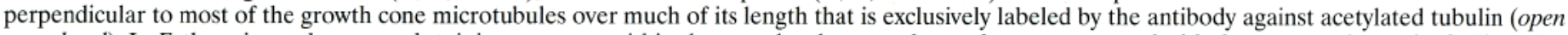

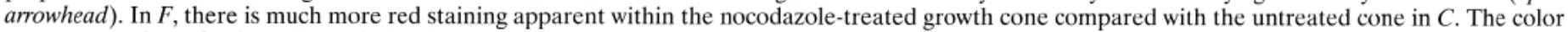
spectrum to the right shows how pixel values were mapped to colors (black was mapped to magenta, white to orange). Scale bar, $5 \mu \mathrm{m}$. 
cone. In untreated growth cones, a subset of the microtubules present in the base of the cone extends into the peripheral domain (P-domain), and the segments of these microtubules that are present within the $\mathrm{P}$-domain are always high in tyrosinated tubulin and are presumably not as stable as more proximal segments (Edson et al., 1993). The P-domain portions of these microtubules were quickly eliminated after acute nocodazole treatment and were not found in growth cones of neurons plated in nocodazole. Although establishing a nocodazole-induced reduction in dynamic instability of the microtubules with plus ends in the growth cone will require measurements of polymerization kinetics of fluorescently tagged microtubules (Lim et al., 1990), the evidence discussed above supports this hypothesis.

How might a decrease in dynamic instability cause the changes in axon assembly that we documented? Variability in the kinetics of microtubule assembly could have an important role in regulating axon assembly. A rapid prolonged polymerization of one or a small subset of the microtubules near the growth cone could provide a scaffolding along which endoplasmic reticulum and other elements of differentiated axoplasm could advance. The distal ends of microtubules in growth cones are often associated with such elements (Dailey and Bridgman, 1989, 1992). This could induce cytoskeletal reorganization that facilitates further axoplasmic differentiation. In some instances, these microtubules would undergo catastrophic shortening, stranding the cargo, but the foray may still accelerate axonal differentiation in that region. Such a role for microtubules that extend into the P-domain is supported by work in invertebrate systems in which progressive recruitment of microtubule plus ends toward a filopodium or a region of a lamellipodium results in progressively more rapid axonal differentiation along the axis of those microtubules (Sabry et al., 1991; Lin and Forscher, 1993). Depolymerization of axonal microtubules could accelerate axonal differentiation indirectly, by increasing the free tubulin concentration in the vicinity of the growth cone, thereby increasing the frequency of rapid prolonged polymerization events of the remaining microtubules. Thus, an agent that decreases dynamic instability could markedly reduce the rate of axon extension by eliminating rapid polymerization events of the distal-most population of microtubules. The decreased rate of catastrophe may not only decrease the free tubulin available for polymerization, but could increase the number of microtubulcs per cross-section.

The above model does not incorporate a mechanism by which nocodazole could influence axon extension by altering the dynamic instability of microtubules in proximal axon segments. Dynamic instability is a stochastic parameter, resulting in only a subpopulation of microtubule plus ends within a given axon segment lengthening or shortening. Because differentiation of the proximal axon is complete, and because it is unlikely that the subpopulation of lengthening microtubules could exert a significant "push" on more proximal structures, it is difficult to imagine how altering dynamic instability in proximal portions of the axon would have an effect on axon extension under normal conditions. Our model predicts that application of nocodazole to the growth cone will be as effective as bath application of nocodazole, and that application of nocodazole to proximal segments alone will have a long latency before generating an effect, corresponding to the time required for transport or diffusion of the nocodazole to the growth cone. This prediction is consistent with the results that were obtained on axon outgrowth using focal application of drugs that affect microtubule stability (Bamburg et al., 1986).

A similar model to ours was suggested by Letourneau's group based on their observations of taxol treated neurons (I etourneau and Ressler, 1984). Although taxol and nocodazole have opposite effects on plus end polymerization of microtubules, taxol has also been shown to reduce dynamic instability of microtubulcs (Jordan et al., 1993). Like nocodazole, taxol causes a reduction in axon elongation rate and an increase in axon diameter. In combination with our results, these data suggest that axon extension rate and diameter are regulated by dynamic instability.

\section{REFERENCES}

Ahmad FJ, Baas PW (1995) Microtubules released from the neuronal centrosome are transported into the axon. J Cell Biol 108:2761-2769.

Baas PW, Ahmad FJ (1992) The plus ends of stable microtubules are the cxclusive nucleating structurcs for microtubules in the axon. J Cell Biol 116:1231-1241.

Baas PW, Ahmad FJ (1993) The transport propertics of axonal microtubules establish their polarity orientation. J Cell Biol 120:1427-1437.

Baas PW, Black MM (1990) Individual microtubules in the axon consist of domains that differ in both composition and stability. $J$ Cell Biol 111:495-509.

Baas PW, Slaughter T, Brown A, Black MM (1991) Microtubule dynamics in axons and dendrites. J Neurosci Res 30:134-153.

Bamburg JR, Bray D, Chapman K (1986) Assembly of microtubules at the tip of the growing axons. Nature 321:788-790.

Bray D, Bunge MB (1981) Serial analysis of microtubules of cultured rat sensory neurons. J Neurocytol 10:589-605.

Brown B, Li Y, Slaughter T, Black MM (1993) Composite microtubules of the axon: quantitative analysis of tyrosinated and acetylated tubulin along individual axonal microtubules. J Cell Sci 104:339-352.

Bridgman PC, Dailey ME (1989) The organization of myosin and actin in rapid frozen nerve growth cones. J Cell Biol 108:95-109.

Dailey ME, Bridgman PC (1989) Dynamics of the endoplasmic reticulum and other membranous organelles in growth cones of cultured neurons. J Neurosci 9:1897-1909.

Dailey ME, Bridgman PC (1992) Structure and organization of membrane organelles along distal microtubule segments in growth cones. I Neurosci Res 30:242-258.

De Brabander MJ, Van de Veire RML, Aerts FEM, Borgers M, Janssen PAJ (1976) The effects of methyl [5-(2-thienylcarbonyl) ${ }^{1} \mathrm{H}-$ benzimidazol-2-yl]carbamate (R 17934; NSC 238159), a new synthetic antitumoral drug interfering with microtubules, on mammalian cells cultured in vitro. Cancer Res 36:905-916.

Edson KJ, Lim S-S, Borisy GG, Letourneau PC (1993) FRAP analysis of the stability of the microtubule population along the neurites of chick sensory neurons. Cell Motil Cytoskeleton 25:59-72.

Gelfand VI, Bershadsky AD (1991) Microtubule dynamics: mechanism, regulation, and function. Annu Rev Cell Biol 7:93-116.

Gordon-Weeks PR, Lang RDA (1988) The $\beta$-tubulin of the growth cone is predominantly in the tyrosinated form. Dev Brain Res 42:156-160.

Gundersen GG, Bulinsky JC (1984) Distinct populations of microtubules: tyrosinated and nontyrosinated alpha tubulin are distributed differently in vivo. Cell 38:779-789.

Hirokawa N (1993) Axonal transport and the cytoskeleton. Curr Opin Neurobiol 3:724-731.

Hirokawa N (1994) Microtubule organization and dynamics dependent on microtubule-associated proteins. Curr Opin Cell Biol 6:74-81.

Jordan MA, Thrower D, Wilson L (1992) Effects of vinblastine, podophyllotoxin and nocodazole on mitotic spindles: implications for the role of microtubule dynamics in mitosis. J Cell Sci 102:401-416.

Jordan MA, Wilson L (1990) Kinetic analysis of tubulin exchange at microtubule ends at low vinblastine concentrations. Biochemistry 29:2730-2739.

Jordan MA, Toso RJ, Thrower D, Wilson L (1993) Mechanism of mitotic block and inhibition of cell proliferation by taxol at low concentrations. Proc Natl Acad Sci USA 90:9552-9556.

Joshi HC, Baas PW (1993) A new perspective on microtubules and axon growth [review]. J Cell Biol 121:1191-1196.

Letourneau PC, Ressler AH (1984) Inhibition of neurite initiation and growth by taxol. J Cell Biol 98:1355-1362.

Li Y, Black MM (1996) Microtubule assembly and turnover in growing axons. J Neurosci 16:531-544. 
Lim S-S, Sammak PJ, Borisy GG (1989) Progressive and spatially differentiated stability of microtubules in developing neuronal cells. J Cell Biol 109:253-263.

Lim S-S, Edson KJ, Letourneau PC, Borisy GG (1990) A test of microtubule translocation during neurite elongation. J Cell Biol 111:29-44.

Lin C-H, Forscher P (1993) Cytoskeletal remodeling during growth cone-target interactions. J Cell Biol 121:1369-1383.

Liao G, Nagasaki T, Gundersen GG (1995) Low concentrations of nocodazole interfere with fibroblast locomotion without significantly affecting microtubule level: implications for the role of dynamic microtubules in cell locomotion. J Cell Sci 108:3473-3483.

Mitchison T, Kirschner M (1984) Dynamic instability of microtubule growth. Nature 312:237-242.
Okabe S, Hirokawa N (1992) Differential behavior of photoactivated microtubules in growing axons of mouse and frog neurons. J Cell Biol 117:105-120

Pryer NK, Walker RA, Skeen VP, Bourns BD, Soboeiro MF, Salmon ED (1992) Brain microtubule-associated proteins modulate microtubule dynamic instability in vitro. J Cell Sci 103:956-976.

Sabry JH, O'Connor TP, Evans L, Toroian-Raymond A, Kirschner M, Bentley D (1991) Microtubule behavior during guidance of pioneer neuron growth cones in situ. J Cell Biol 115:381-395.

Tanaka E, Kirschner MW (1991) Microtubule behavior in the growth cones of living neurons during axon elongation. J Cell Biol 115:345-363.

Tanaka E, Ho T, Kirschner MW (1995) The role of microtubule dynamics in growth cone motility and axonal growth. J Cell Biol 128:139-155. 